\title{
Fingerprinting used to combat drug-resistant TB in India, Cambodia
}

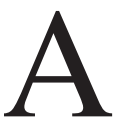

$\mathrm{s}$ India copes with the growing problem of drug-resistant tuberculosis, a nongovernmental organization is making headway in encouraging people to take their medication by linking counselling with fingerprinting technology.

In more than 2000 communities in India and Cambodia, Operation ASHA has equipped high school-educated tuberculosis (TB) counsellors with biometric scanning equipment and laptops intended to make it easier to monitor the medication adherence of people diagnosed with TB.

In these communities, men and women on their way to work stop by a clinic or counsellor's house three times a week, scan their fingerprints and take antibiotics. Those fingerprints are stored in ASHA's system, which tracks them over time. If the program does not receive a fingerprint when the next dose is due, it sends an alert to the counsellor and a supervisor. The counsellor then has 48 hours to track down and persuade patients to take their medication.

Using the technology is an effective means to help prevent the growth of multidrug-resistant and totally drugresistant TB, says Shelly Batra, the president of Operation ASHA, based in New Delhi. Patients are supposed to take their antibiotics for months, but many stop early, which can fuel resistant strains.

Almost 2 million people develop tuberculosis in India every year, and there are almost 100000 cases of drugresistant TB.

"The entire world is going to be in big trouble" if multidrug-resistant and totally drug-resistant tuberculosis spreads, Batra says.

In a 2011 journal article (Clin Infect Dis 2011;154:579-81), Dr. Zarir Udwadia reported four cases that he characterized as totally drug-resistant, and media reports later identified another eight.

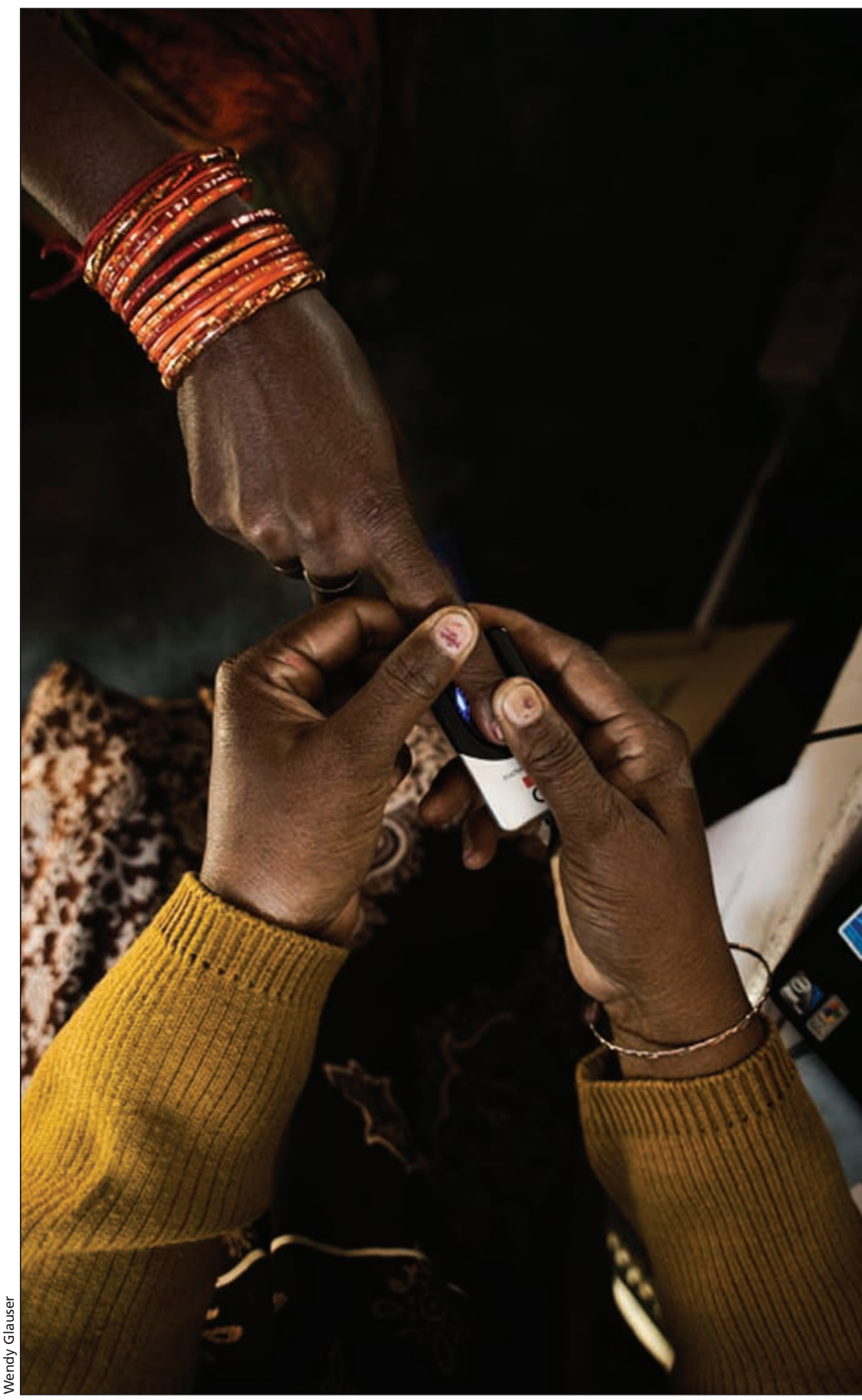

Patients stop by a clinic or counsellor's house three times a week, scan their fingerprints and take antibiotics. If the program does not receive a fingerprint when the next dose is due, it sends an alert to the counsellor and a supervisor. 
Both the World Health Organization (WHO) and the Indian government have rejected the characterization of totally drug-resistant cases, arguing that new drugs are being developed whose effectiveness against these strains has not been tested.

In addition to the loss of life that would result if resistant tuberculosis spread, Batra points to the anticipated economic losses that would result, citing a WHO statistic that treating 1.3 million drug-resistant TB cases between 2010 and 2015 will cost US\$ 16 billion. "There's going to be tremendous economic loss, [and] millions will be wiped out," Batra says.

Studies have demonstrated the effectiveness of directly observed therapy short course (DOTS) strategy, under which counsellors are paid to ensure patients with TB take their medications so they don't develop resistance. Counsellors are paid more based on the number of adherent patients they oversee, a practice that encourages the counsellors to locate patients who haven't shown up to take their medication on time. But without fingerprinting, counsellors sometimes recorded higher numbers of compliant patients than they actually saw, in order to receive their bonus. On several occasions, auditors discovered the deception, Batra says.

Under ASHA's Microsoft-funded fingerprint system, a counsellor can only register a patient as having taken their drugs with the confirmation of a fingerprint, indicating the patient is physically present at the time.

The technology not only reassures ASHA administrators that patients are indeed following their drug regimen, it also helps counsellors appeal to their patients.

"They tell the patient, 'If you don't come to get the medicine, I will be micro-monitored by the technology device and if there are too many defaulters, I could lose my job'," says Batra. She says the technology has reduced the defaulter rate.

Operation ASHA has already treated 30000 patients using the new technology, and only 750 of them have defaulted. The customary default rate is as high as $36 \%$, according to Batra, meaning as many as 10800 patients might have stopped taking their medication without ASHA's program.

Although ASHA is the first organization to use fingerprint technology for TB treatment, organizations have used it to assist patients who are taking antiretroviral agents, says Batra.
Dr. Walter Curioso, general director of statistics and informatics at the Ministry of Health in Peru, says biometric identification saves lives and will become increasingly common as other governments tie national identity databases to fingerprints, as Peru began to do in 2007.

Fingerprints are now relied upon to identify patients in emergency rooms in major Peruvian hospitals, says Curioso. "You can identify people that are unconscious," he says. "You can know if the person is allergic to some medicine."

Moreover, fingerprint systems can avoid misidentification and stop patients from doubling up on or missing interventions. This is particularly pertinent in developing countries, which tend to have complex government, charity and private health care provider networks, Curioso adds.

Patients in the United Kingdom have resisted fingerprinting technology because of Big Brother and privacy concerns, but ASHA's patients so far appear unconcerned about the scanners. "TB patients don't like photographs, but a fingerprint is okay," Batra says. Wendy Glauser, New Delhi.

CMAJ 2013. DOI:10.1503/cmaj.109-4470 